\title{
Validação do Teste Need For Cognition: Um Estudo em Contabilidade Comportamental
}

Validation of the test need for cognition:

A study in behavioral accounting

Validación de la prueba need for cognition: Un estudio en contabilidad comportamental

Raimundo

Nonato Lima Filho,

Adriano Leal Bruni \&

Igor Gomes Menezes

Universidade Federal da Bahia

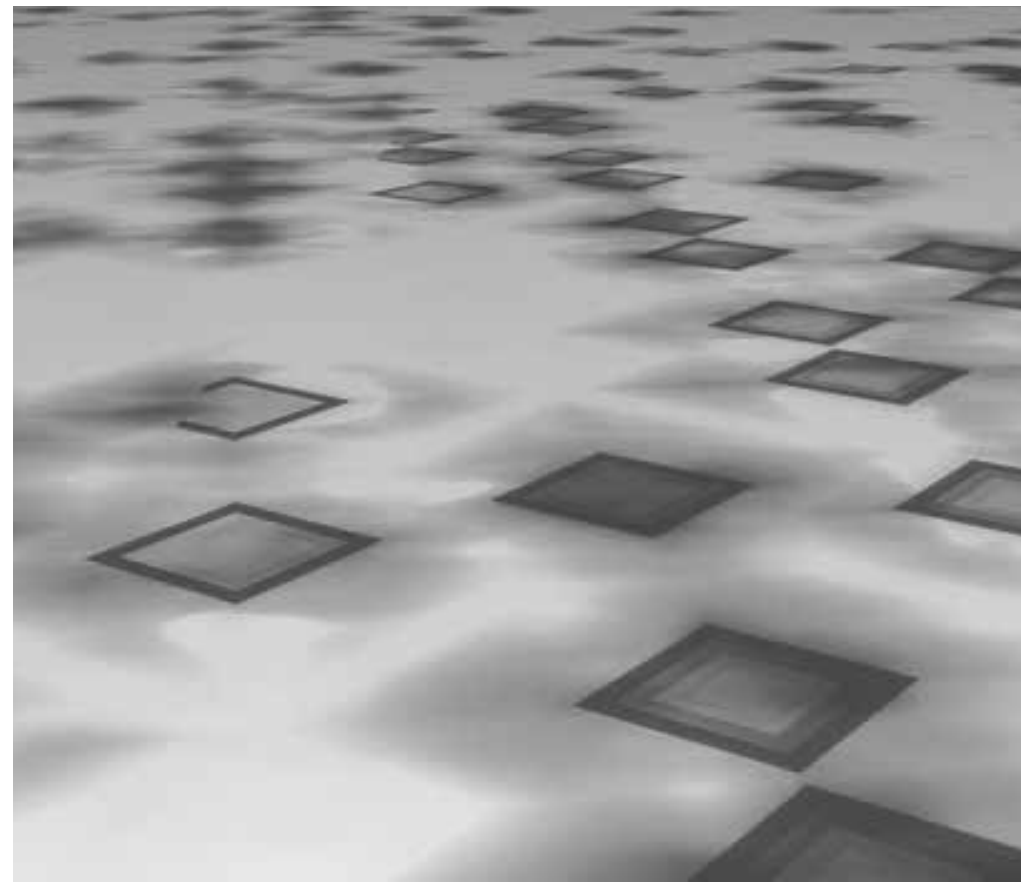


Resumo: Este estudo teve como objetivo validar a escala Need For Cognition (NFC) em estudos em Contabilidade comportamental. Ademais, buscou-se medir possíveis correlações entre o nível de necessidade de cognição e a existência de vieses cognitivos em decisões contábeis e financeiras. Para efetivar o processo de validação completo, foram realizadas duas validações - critério e construto. A análise foi feita mediante a apreciação de uma amostra formada por 128 estudantes de pós-graduação. A técnica estatística utilizada para validação desse teste foi a análise fatorial, por possuir a capacidade de determinar o grau de influência de determinada variável na explicação de um fator, e, para o tratamento dos dados, foi utilizada a regressão logística por possibilitar a explicação de valores em função de valores conhecidos ou de variáveis independentes. Os resultados da validade de construto apontaram a legitimidade do NFC como escala unidimensional, excluindo-se três outputs de sua escala original; já os resultados da validade de critério confirmaram o impacto do nível de cognição na maximização da ocorrência de heurísticas em decisões gerenciais.

Palavras chave: Validade do teste. Cognição. Análise do comportamento. Psicologia cognitiva.

Abstract: This study aimed to validate the Need for Cognition scale (NFC) in behavioral accounting. In addition, we sought to measure the possible correlations between the level of need for cognition and the existence of cognitive biases in decisions in accounting and financial information. Two validations were performed to carry out the process of full validation - criterion and construct. The analysis was done by the examination of a sample comprised by 128 graduation students. The statistical technique used for the validation of this test was a factorial analysis for it has the ability to determine the degree of influence of a particular variable in the explanation of a factor, and the processing logistic regression was used for the explanation of possible values as a function of known values or independent variables. The results of the construct of validity showed the legitimacy of the NFC as a unidimensional scale excluding three outputs of its original scale, since the criterion validity of the results confirmed the impact of the level of cognition in maximizing the occurrence of heuristics in managerial decisions.

Keywords: Test validity. Cognition. Behavioral assesment. Cognitive psychology.

Resumen: Este estudio tuvo como objetivo validar la escala Need For Cognition (NFC) en estudios en Contabilidad comportamental. Además, se buscó medir posibles correlaciones entre el nivel de necesidad de cognición y la existencia de planteos cognoscitivos en decisiones contables y financieras. Para hacer efectivo el proceso de validación completo, fueron realizadas dos validaciones - criterio y constructo. El análisis fue realizado mediante la apreciación de una muestra formada por 128 estudiantes de posgrado. La técnica estadística utilizada para la validación de esa prueba fue el análisis factorial, por poseer la capacidad de determinar el grado de influencia de determinada variable en la explicación de un factor, y, para el tratamiento de los datos, fue utilizada la regresión logística por posibilitar la explicación de valores en función de valores conocidos o de variables independientes. Los resultados de la validez de constructo apuntaron la legitimidad del NFC como escala unidimensional, excluyéndose tres outputs de su escala original; ya los resultados de la validez de criterio confirmaron el impacto del nivel de cognición en la maximización de la ocurrencia de heurísticas en decisiones gerenciales.

Palabras clave: Validación del test. Cognicion. Analisis de la conducta. Psicología cognitiva.

O principal objetivo desta pesquisa é a validação, no contexto brasileiro e da Contabilidade comportamental, de um instrumento psicométrico para a mensuração da necessidade de cognição. Adicionalmente, o trabalho objetiva analisar eventuais erros de julgamento em situações caracterizadas no âmbito na Contabilidade, estudados a partir da necessidade de cognição do indivíduo. Desse modo, este estudo busca encontrar evidências acerca do impacto que essa variável exerce, minimizando a presença de heurísticas, por meio da resposta ao seguinte problema de pesquisa: de que forma a cognição de um indivíduo afeta a ocorrência de heurísticas em decisões gerenciais?

O ambiente organizacional moderno é marcado pela necessidade de compreensão de um conjunto complexo de variáveis e relações. A empresa opera em um cenário caracterizado pela incerteza e por mercados 
imperfeitos. Dentro desse contexto, cabe à Contabilidade compreender e subsidiar a tomada de decisão e o processo de julgamento de informações, produzindo subsídios relevantes que poderão influenciar nesse processo. Aos conhecimentos contábeis, é preciso incorporar noções oriundas da Economia, da Estatística e da Psicologia cognitiva. A fusão dos conceitos representa a amálgama fundamental dos estudos conduzidos no âmbito da Contabilidade comportamental.

A relação entre variáveis de personalidade e o comportamento do indivíduo é do escopo de diferentes campos do conhecimento incluídos no entendimento do processo pelo qual essas variáveis influenciam a tomada de decisão. As pesquisas que envolvem a Contabilidade comportamental visam à probabilidade de segmentar uma decisão de acordo com as perspectivas individuais, seja através das confianças particulares, seja das diferenças de personalidade ou de estilos em relação a aspectos específicos.

Contrapondo a máxima utilização da racionalidade no processo de julgamento defendido pela hipótese neoclássica do agente econômico (homem econômico), Simon (1955) admite a ideia de uma racionalidade limitada, na qual o processo de julgamento é feito a partir de uma realidade simplificada, considerando apenas as informações que são realmente relevantes nesse processo.

Em um ambiente organizacional, ao contrário do que acreditam as teorias clássicas, a representação moderna aponta um conjunto de variáveis e de relações, nas quais a empresa opera em um cenário complexo, caracterizado principalmente por uma situação de incerteza e por um mercado imperfeito. Dentro desse contexto, cabe à ciência contábil subsidiar a tomada de decisão dessas organizações, produzindo as informações relevantes que poderão influenciar nesse processo. Todavia, para maximizar essa contribuição, associaram-se aos conhecimentos contábeis noções oriundas da Economia, da Estatística e da Psicologia cognitiva.

Essa segmentação pode ser entendida utilizando-se ferramentas pertinentes que assistem na caracterização de uma pessoa. As decisões podem ser diversas, mesmo quando são submetidas a estímulos análogos.

De acordo com o modelo da probabilidade da elaboração (ELM), existem dois aspectos de persuasão: o central, por meio do exame racional e da apreciação dos argumentos, e o periférico, fundamentado em simples parecer afetivo, que desconsidera a análise cognitiva. Empregando o aspecto central, o tomador de decisão emprega a informação relevante e, adotando o aspecto periférico, utiliza exclusivamente simples alusões ou influência do contexto.

Na literatura da Psicologia cognitiva, existem diversos instrumentos que mensuram a necessidade de cognição. Entre eles, está o NFC (Need for Cognition). Esse instrumento foi desenvolvido por Cacioppo, Petty e Kao (1984); a configuração de questionário possui 18 outputs, e a escala empregada nessa pesquisa é de sete pontos, com variação de 1 (discordância muito intensa) a 7 (concordância muito intensa). A soma dos pontos para os 18 itens rotula o respondente como baixa necessidade de cognição ou alta necessidade de cognição. Segundo Haugtvedt, Petty e Cacioppo (1992), indivíduos com alto NFC empregam o aspecto central, ou seja, a capacidade de analisar os argumentos mais exaustivamente; por sua vez, os indivíduos com baixo NFC, que utilizam o aspecto periférico, estimam perspectivas marginais. Pesquisas sobre comportamento humano permitiriam aperfeiçoar teorias que corroboraram a perspectiva racional que envolve a tomada de decisão. No entanto, 
poucas pesquisas científicas foram realizadas nessa linha de pesquisa. No Brasil, os estudos sobre Contabilidade comportamental são escassos (Milanez, 2003) e praticamente inexistentes sobre o comportamento do tomador de decisão em práticas gerenciais. Melhor entendimento e delimitação das falhas cognitivas individuais possibilitariam aos gestores melhorarem sua capacidade decisória, evitando falhas nesse processo.

A relevância desta pesquisa está justamente em contribuir com subsídios teóricos e estudos em Contabilidade comportamental, principalmente na compreensão do gestor envolvido em decisões gerenciais.

\section{Referencial teórico}

A validação é o método de analisar a exatidão de uma inferência concretizada a partir das escalas de um teste psicométrico. Validar não é só demonstrar o valor de um instrumento de medida, mas todo um processo de investigação. A validação não se esgota, ao contrário, implica prosseguimento e deve ser repetida quantas vezes forem necessárias para seu definitivo entendimento. Segundo Raymundo, "valida-se não propriamente o teste, mas a interpretação dos dados decorrentes de um procedimento específico" (2009, p. 87).

As dificuldades que as pessoas têm de julgar subjetivamente probabilidades, de analisar e de processar informações para posteriormente tomarem decisões advém de um processo denominado ilusão cognitiva (Kahneman \& Riepe, 1998). Segundo essa perspectiva, a ilusão cognitiva é a tendência em cometer erros sistemáticos na tomada de decisão. No trabalho de Kahneman e Tversky (1979), tais ilusões são classificadas como heurísticas no processo decisório e causadas pela escolha de determinados procedimentos mentais apontados pela teoria dos prospectos. As heurísticas podem ser entendidas como simplificações mentais que provocam distorções na tomada de decisões. Já viés cognitivo, para Kivetz, "refere-se ao conjunto de operações cognitivas usadas por indivíduos para organizar, avaliar e acompanhar atividades financeiras" (1999, p. 249).

O fenômeno da heurística tem sido muito estudado no campo da Filosofia e das ciências, com os trabalhos de Fenwick e Cheryl (2008), Jeon e Shyam (2010) e Subhashis, Subhas e Bhargab (2010). As heurísticas, nessas áreas, são estudadas sob os conceitos de Lakatos, que utilizam a perspectiva das MSRP - metodologias dos programas de investigação científica.

Tversky e Kahneman (1974), em seu artigo Judgement under Uncertainty: Heuristics and Biases, constataram, por meio de múltiplos experimentos, que as pessoas, perante situações em que necessitariam avaliar e escolher a alternativa que pareceria mais correta, basearam essas escolhas em um número limitado de princípios heurísticos, que reduzem a complexidade das atividades de avaliar e de prever valores, tornando-as operações de julgamento mais simples, utilizando, dessa forma, as heurísticas.

Pode-se afirmar que, no ambiente empresarial, em um mercado globalizado cada vez mais competitivo, busca-se tomar decisões mais rápidas, corretas e abrangentes. As decisões visam a minimizar perdas, a maximizar ganhos e a criar uma situação em que, comparativamente, o decisor acredite na obtenção de ganhos entre o estado da natureza em que se encontrava e o que irá encontrar após implantar o julgamento. O teste NFC foi elaborado por Cohen, Stotland e Wolfe (1955), e nasceu dos estudos da personalidade e da Psicologia social (Sarnoff \& Katz, 1954 como citado em Cacioppo \& Petty, 1982), Para esses autores, o NFC é "a necessidade de estruturar 
situações relevantes de forma integrada e com sentido, de compreender e tornar lógico o mundo experiencial". Cacioppo e Petty fazem uso do conceito como modo de conhecer indivíduos que têm interesse na preparação criteriosa da informação. O conceito passa, assim, a determinar uma característica de personalidade (motivação intrínseca), definida como o grau em que o indivíduo se envolve em atividades cognitivas e tem gosto em realizar essas atividades. Cacioppo, Petty, Feinstein e Jarvis (1996) afirmam que os indivíduos com elevados níveis de necessidade de cognição "tendem naturalmente a procurar, adquirir e refletir sobre a informação de forma a dar sentido aos estímulos processados".

Em comparação, indivíduos com níveis baixos de necessidade de cognição têm "mais chance de acreditarem nos outros, em heurísticas cognitivas, ou em processos de comparação social para fornecer essa estrutura" (Cacciopo et al., 1996). Não é que sejam diferenciados como inábeis para verificar a informação minuciosamente, mas habitualmente optam por evitar esforço cognitivo (Haugtvedt, Petty, \& Cacioppo, 1992).

Pesquisas têm indicado que as pessoas com alta necessidade de cognição consideram histórias ambíguas como menos aprazíveis que histórias com estrutura organizada (enquanto essa discriminação não foi expressiva em pessoas com baixa necessidade de cognição) (Cohen, Stotland, \& Wolfe, 1955) e alegam gostar mais de um trabalho quando este demanda regras difíceis em vez de regras simples, enquanto pessoas com necessidades de cognição menos expressivas escolhem os serviços com princípios simples (Cacioppo \& Petty, 1982), suscitam mais pensamentos coerentes com a mensagem e pensamentos mais adequados em relação aos contextos fortes (Cacioppo, Petty, \& Morris, 1983), buscam mais conhecimento em tomadas de decisão (Verplanken, Hazenberg, \& Palenewen, 1992), esforçam-se mais em processamentos de esforço em comunicações persuasórias (Cacioppo, Petty, Kao, \& Rodriguez, 1986; Haugtvedt et al., 1992), destinam mais pensamentos relevantes a um assunto ou a determinada matéria que os indivíduos com baixa necessidade de cognição (Cacioppo et al., 1983; Haugtvedt et al., 1992), aspiram a adotar uma direção mais analítica e sistemática elegendo contextos fortes a fracos (Cacioppo \& Petty, 1983), acreditam mais na propriedade e na coerência da informação e são mais entusiastas por esses fatores, enquanto indivíduos com baixa necessidade de cognição tendem a acreditar em pistas heurísticas para analisar as mensagens e são mais entusiasmados por essas pistas (Axsom, Yates, \& Chaiken, 1987; Haugtvedt et al., 1992).

Para os indivíduos com baixa necessidade de cognição, apresentar uma dificuldade seguida da sua resposta gera maior mudança de atitude do que apresentar a resposta seguida do problema que a gerou (apesar disso, as pessoas com alta necessidade de cognição não fizeram essa discriminação, pois sentiamse mais determinadas para pensar sobre o problema, e a dissolução independe da sua apresentação) (Cohen, 1957 como citado em Cacioppo \& Petty, 1984), provocam menos opiniões em um serviço de brainstorming quando o encargo da tarefa era conferido ao grupo do que quando a responsabilidade era somente conferida ao próprio, enquanto as pessoas com alta necessidade de cognição suscitam o mesmo número de opiniões nas duas categorias (Petty, Cacioppo, \& Kramer, 1985 como citado em Cacioppo et al., 1986) e davam prioridade a itens que faziam citação a heurísticas em vez dos itens que faziam alusão a um processamento que promovia esforço e atenção, enquanto as pessoas com alta necessidade de cognição davam prioridade a itens que se referiam a um processamento que promovia esforço e atenção em vez de itens com menção a heurísticas (Chaiken, 1987), acreditam mais em um manancial atrativo 


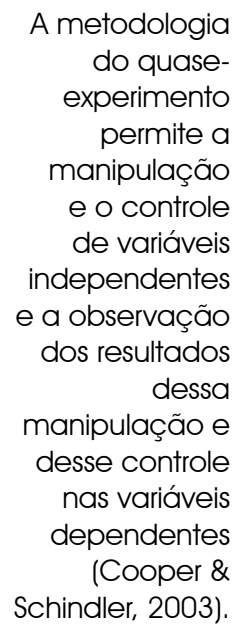

do que nos não atrativos (Cacioppo \& Petty, 1983), e também em pistas simplórias para aperfeiçoar e transformar as suas atitudes (Cacioppo et al., 1983).

Assim, entende-se o NFC como uma tendência/ teste de procurar no processamento cognitivo um rigoroso processo de conhecimento. É uma escala que foi desenvolvida para avaliar as diferenças individuais, e, dessa forma, representa a tendência do indivíduo em se engajar em atividades que exijam esforço analítico (Cacioppo \& Petty, 1982 como citado em Deliza, Rosenthal, \& Costa, 2003).

\section{Procedimentos metodológicos}

Ao fazer parte de um estudo mais abrangente, em que se buscou investigar a existência de relações entre diferentes variáveis, utilizou-se o desenho da pesquisa quaseexperimental. Para se alcançar os objetivos desta pesquisa, foi necessário investigar a existência de relações entre algumas variáveis, o que dependeu do controle de fatores que pudessem intervir nos resultados e mascarar a alteração das variáveis apresentadas em um único tipo de questionário. A metodologia do quase-experimento permite a manipulação e o controle de variáveis independentes e a observação dos resultados dessa manipulação e desse controle nas variáveis dependentes (Cooper \& Schindler, 2003).

Nesta pesquisa, adota-se a validade de construto e a validade de critério para atender aos objetivos traçados. A validade de construto está vinculada ao objetivo central (validar o teste NFC em Contabilidade comportamental), e esse tipo de validade permite definir qual a atributo que explica a variância do teste. A validade de construto refere-se à comprovação de que o instrumento empregado realmente afere aquilo que se indica aferir. Conforme Raymundo, as evidências indispensáveis para a validação de construto "são obtidas fazendo-se uma série de estudos inter-relacionados, por meio de testes estatísticos, das construções teóricas sobre a relação entre as variáveis a serem medidas" (2009, p. 88).

Já a validade de critério está vinculada ao objetivo secundário deste estudo (medir possíveis correlações entre o nível de necessidade de cognição e a existência de vieses cognitivos em decisões contábeis e financeiras), e esse tipo de validade está relacionado a um fator que, todavia, pode receber a influência de outros fatores que não estão integrados à variável principal, o que pode alterar a amplitude do coeficiente de validade. Raymundo afirma que a validade de critério analisa a "correlação entre os escores do teste $(X)$ e os escores da variável critério (Y)" (2009, p.88), portanto, nesta pesquisa, analisa-se a correlação dos escores do teste NFC (X) e os escores das heurísticas apresentadas nos cenários propostos $(\mathrm{Y})$, corroborando assim a utilização dos dois tipos de validades.

O estudo da eventual influência do nível de necessidade de cognição na presença de heurísticas em cenários gerenciais foi realizado através da análise de uma amostra formada por estudantes de pós-graduação de Salvador (BA). A amostra foi composta por alunos de IES públicas e privadas, escolhidos entre diferentes cursos de doutorado, mestrado ou especialização, que envolvem, propositalmente, cursos relacionados com as áreas de Administração, finanças ou Contabilidade, e não relacionados a essas áreas. O uso de uma amostra diversificada permitiu segregar os respondentes em função do seu variado nível de envolvimento com decisões gerenciais e agrupá-los em dois níveis distintos.

A hipótese de pesquisa foi criada e testada a partir da efetivação de um modelo operacional de pesquisa. As variáveis empregadas foram necessidade de cognição e heurísticas, conforme apresentado na Figura 


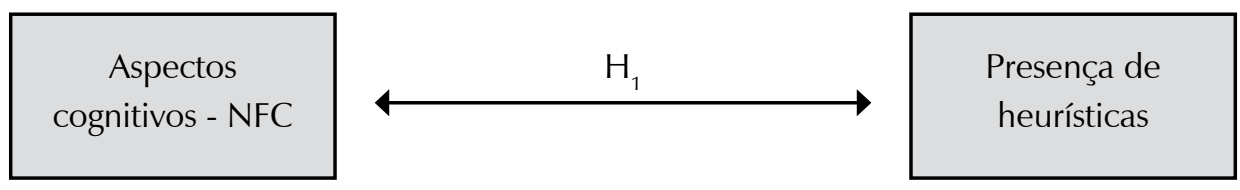

Figura 1. Modelo operacional da pesquisa

Fonte: Elaboração própria (2011)

\section{Procedimentos de coleta de dados}

A análise da eventual presença de heurísticas em decisões gerenciais foi feita mediante a análise de uma amostra formada por 128 estudantes de pós-graduação de Salvador, BA, com coleta de dados ocorrida em setembro de 2010. A amostra foi composta por alunos de IES públicas e privadas, escolhidos entre diferentes cursos de doutorado, mestrado ou especialização, que envolviam, propositalmente, cursos relacionados à Administração e à Contabilidade (doutorado em Administração, mestrado acadêmico em Administração, especialização em Auditoria FiscoContábil, especialização em Gestão Tributária, especialização em Contabilidade Gerencial e MBA em Finanças Empresariais), e não relacionados (doutorado em Difusão do Conhecimento, mestrado em Educação, mestrado em Gestão Social e Desenvolvimento e especialização em Gestão de Projetos).

O tamanho da amostra seguiu a sugestão de Hai, Anderson, Tatham e Black (1998), com não menos que 30 sujeitos por célula de pesquisa, com as células apresentando quantidade próxima de observações.

Pesquisas realizadas por Liyanarachchi e Milne (2005) e Elliott, Hodge, Kennedy e Pronk (2007) sugerem que estudantes, seja de graduação ou de pós-graduação, podem ser utilizados em pesquisas acadêmicas, pois representam bons substitutos para as pesquisas realizadas com profissionais.

A análise da graduação dos respondentes indicou que 25\% eram graduados em Contabilidade, aproximadamente 33\% em Administração e 42\% apresentavam outras graduações. Como um dos objetivos do estudo foi comparar resultados de profissionais com alto e baixo envolvimento com decisões gerenciais - o que seria a característica mais frequente em profissionais de Administração e Contabilidade - a dispersão da graduação dos respondentes é coerente e desejada. Em relação ao gênero, cerca de $48 \%$ dos respondentes eram do sexo feminino e $52 \%$, aproximadamente, do sexo masculino. Em relação à idade, 50\% dos respondentes apresentavam entre 24 e 30 anos.

\section{Teste de hipóteses}

Para responder ao questionamento desta pesquisa, foi proposta uma hipótese com a finalidade de conduzir a verificação empírica dos objetivos definidos em alinhamento ao problema a ser pesquisado.

A hipótese, aqui denominada $\mathrm{H}_{1}$, sustenta que, quanto menor for o nível de cognição dos respondentes, maior será a presença de heurísticas. 
Em pesquisa recente realizada na Alemanha, publicada na Personality and Social Psychology Bulletin, os autores concluíram que a inteligência de um adulto não pode ser plenamente compreendida sem considerar os fatores cognitivos, e que vieses e nível de cognição são grandezas inversamente proporcionais (Fleischhauer et al., 2010).

Para poder testar as hipóteses, foram desenvolvidos dois blocos de questões, apresentados em um único tipo de questionário. No primeiro bloco, apresentamse cenários com possíveis heurísticas inseridas, solicitando ao respondente algum julgamento. O segundo bloco apresenta o teste NFC Need for Cognition, que foi desenvolvido por Cacioppo, Petty e Kao (1984), traduzido e validado por Deliza, Rosenthal e Costa (2003), e que foi apresentado sem o rótulo a fim de que os respondentes não soubessem que estavam testando seu nível de necessidade de cognição, evitando assim vieses nas respostas.

\section{Instrumentos de pesquisa}

O primeiro bloco do instrumento de pesquisa consiste na apresentação de seis situações distintas. Em todos os cenários, um potencial viés de heurística foi inserido. Os cenários foram construídos seguindo a indicação de Hansen e Helgeson (1996) e Pohl (2006), que aplicaram testes empíricos para mensurar o nível de heurísticas nas perspectivas de suas pesquisas. Esses autores trabalharam com potenciais heurísticas em simulações nas quais o respondente deveria apresentar sua posição diante daquele cenário. Em seguida, os pesquisadores avaliaram como as respostas se comportaram e se a heurística inserida realmente influenciou seu julgamento. Nesta pesquisa, esse primeiro bloco está alinhado a essa finalidade, em que heurísticas inseridas poderão influenciar a resposta do aluno pesquisado. A seguir, apresentam-se as perspectivas de cada cenário, e qual o comportamento esperado em cada heurística inserida.
$\mathrm{Na}$ primeira situação (a), questiona-se a percepção do respondente em relação à lucratividade de um supermercado brasileiro, com margem de lucro bruto igual a $25 \%$. Para manifestar sua opinião, o respondente deveria escolher 0 (pouco lucrativo) ou 1 (muito lucrativo). A eventual heurística ou ancoragem (predição numérica feita a partir de valor inicial disponível) poderia estar na apresentação da margem de lucro bruto de empresas de telefonia norueguesas, apresentada como igual a $7 \%$. Em uma perspectiva racional, o negócio de supermercados no Brasil não guarda nenhuma relação com o negócio de telefonia na Noruega; assim, racionalmente analisando, não deveria existir qualquer possibilidade de comparação da performance dos supermercados brasileiros com base no desempenho das telefônicas norueguesas. Porém, caso a ancoragem se manifestasse, o respondente julgaria o desempenho dos supermercados nacionais com base nas empresas telefônicas estrangeiras, escolhendo a opção muito lucrativo. Para se confirmar a presença da ancoragem no respondente, apresentamos uma situação análoga na situação (e). Nesse cenário, uma empresa varejista fixou em $10 \%$ a margem de seu lucro líquido, e a eventual heurística inserida está no fato da vivência de um membro da equipe que elabora o orçamento, afirmando que uma indústria em que ele trabalhou durante 20 anos fixava sua margem de lucro líquido em 30\%. Assim, racionalmente analisando, não deveria existir qualquer possibilidade de comparação das empresas de comércio varejista com base na empresa do setor industrial. Porém, caso a ancoragem se manifestasse, o respondente julgaria o desempenho da empresa varejista com base nas empresas industriais, escolhendo a opção pouco lucrativa.

A segunda situação (b) fala da divisão aleatória de amostra de margens de lucro de lojas de material de construção em duas partes. Nada era dito sobre o tamanho dessa amostra. 
Em uma análise puramente racional, há de se imaginar que o que ocorra em uma metade também ocorra na outra. Assim, a média da segunda metade deveria ser aproximadamente igual à média da primeira metade. Como os números da primeira metade foram iguais a $15 \%$, um respondente racional deveria apresentar uma estimativa pontual para a média da segunda metade nesse mesmo intervalo. A situação, porém, apresentava uma potencial heurística, quando dizia que a primeira empresa da segunda metade da amostra tinha margem de lucro igual a $2 \%$. Caso a heurística se manifestasse, os respondentes forneceriam estimativas baseadas no comportamento do primeiro elemento da segunda metade da amostra (dentro do intervalo entre 1\% e $5 \%$ ). O fenômeno dessa situação poderia ser simultaneamente classificado como um efeito de ancoragem (predição numérica feita a partir de valor inicial disponível) e de disponibilidade de instâncias ou cenários (a frequência ou estimativa de uma classe ocorreria a partir de um desenvolvimento particular, no caso, o primeiro elemento da segunda metade). O cenário (f) apresenta a mesma dinâmica, desta vez utilizando regionais de uma empresa de chocolates, que deveria reduzir suas vendas.

A terceira situação (c) do experimento comentava a revisão das vendas orçadas de uma mineradora brasileira e questionava a estimativa do respondente para o percentual de redução. Uma eventual heurística era sugerida mediante a informação de que agências de turismo japonesas haviam reduzido a sua previsão de vendas em $5 \%$. Em uma perspectiva puramente racional, imagina-se que as vendas de agências de turismo no Japão não guardem relação com as vendas de uma mineradora nacional. Caso a representatividade (julgamento da probabilidade de um evento ou objeto A pertencer à classe ou processo B) se manifestasse, estimativas menores seriam apresentadas (dentro do intervalo entre $2 \%$ e $8 \%$ ), sofrendo os efeitos da ancoragem e da representatividade; caso contrário, deveria apresentar a situação (d), em que a taxa de investimento em infraestrutura de uma empresa de manutenção industrial está em $55 \%$, e o julgamento da taxa de investimento de uma filial de uma rede de restaurantes deverá ser influenciado por esse percentual. O nível de presença de heurística foi mensurado atribuindo-se 1 (um) ponto para as alternativas com heurísticas inseridas e 0 (zero) ponto para as alternativas sem heurísticas. Considerando que as respostas sejam aleatórias, o valor esperado de cada cenário será 0,5 ponto. Como foram apresentados seis cenários, o valor esperado do conjunto será três pontos $(6 \times 0,5)$. Assim, subtrai-se a nota real do respondente de 3 (três), e obtém-se o escore da presença de heurística. A incorrência em heurística, neste estudo, determinou que níveis negativos apresentam menor presença de heurísticas, que níveis positivos apresentam maior presença de heurística e níveis iguais a 0 (zero) foram expurgados da análise, por configurarem respostas aleatórias. Segundo Meyer (1983), a soma de muitas variáveis independentes aleatórias e com a mesma distribuição de probabilidade sempre tende a uma distribuição normal. Para uma amostra suficientemente, a distribuição de probabilidade da média amostral pode ser aproximada por uma distribuição normal, com média e variância iguais às da população. O segundo bloco do instrumento de pesquisa, conforme a Tabela 1, foi o teste NFC, traduzido e validado por Deliza, Rosenthal e Costa (2003). O desenvolvimento e a validação de uma escala de confiança, que abarcam tanto aspectos cognitivos e afetivos quanto comportamentais, apresentam suma importância tanto para a academia quanto para gestores. Sob a perspectiva acadêmica, o desenvolvimento de medidas para mensuração das confianças cognitiva, afetiva e comportamental e o teste-modelo proposto deve ser visto como um passo inicial para a teorização sobre as relações que se estabelecem entre os três tipos de confiança. 
Tabela 1. Escala Need for Cognition

\begin{tabular}{|c|c|c|c|c|c|c|c|}
\hline \multirow[b]{2}{*}{ (a) Prefiro problemas complexos aos simples. } & \multicolumn{4}{|c|}{$\begin{array}{l}\text { Discordo } \\
\text { Totalmente }\end{array}$} & \multicolumn{3}{|c|}{$\begin{array}{l}\text { Concordo } \\
\text { Totalmente }\end{array}$} \\
\hline & 1 & 2 & 3 & 4 & 5 & 6 & 7 \\
\hline $\begin{array}{l}\text { (b) Gosto de ter a responsabilidade de lidar com situação } \\
\text { que requer muito pensar. }\end{array}$ & 1 & 2 & 3 & 4 & 5 & 6 & 7 \\
\hline (c) Pensar é meu passatempo preferido. & 1 & 2 & 3 & 4 & 5 & 6 & 7 \\
\hline $\begin{array}{l}\text { (d) Antes faria alguma coisa que desafiaria minhas } \\
\text { habilidades em relação ao pensar que alguma coisa que } \\
\text { requer pouco pensar, certamente. }\end{array}$ & 1 & 2 & 3 & 4 & 5 & 6 & 7 \\
\hline $\begin{array}{l}\text { (e) Tento antecipar situações onde exista a provável chance } \\
\text { de ter que pensar profundamente sobre alguma coisa. }\end{array}$ & 1 & 2 & 3 & 4 & 5 & 6 & 7 \\
\hline $\begin{array}{l}\text { (f) Sinto satisfação em ter que ponderar arduamente por } \\
\text { muito tempo. }\end{array}$ & 1 & 2 & 3 & 4 & 5 & 6 & 7 \\
\hline $\begin{array}{l}\text { (g) Apenas me envolvo intensamente quando tenho que me } \\
\text { envolver. }\end{array}$ & 1 & 2 & 3 & 4 & 5 & 6 & 7 \\
\hline $\begin{array}{l}\text { (h) Prefiro pensar nos problemas pequenos do dia a dia que } \\
\text { nos problemas a longo prazo. }\end{array}$ & 1 & 2 & 3 & 4 & 5 & 6 & 7 \\
\hline $\begin{array}{l}\text { (i) Gosto de tarefas que requerem pouco pensar, uma vez } \\
\text { que as tenha aprendido. }\end{array}$ & 1 & 2 & 3 & 4 & 5 & 6 & 7 \\
\hline $\begin{array}{l}\text { (j) A ideia de utilizar pensamentos para me animar me } \\
\text { parece interessante. }\end{array}$ & 1 & 2 & 3 & 4 & 5 & 6 & 7 \\
\hline $\begin{array}{l}\text { (k) Eu realmente gosto de uma tarefa que envolva pensar } \\
\text { em novas soluções para os problemas. }\end{array}$ & 1 & 2 & 3 & 4 & 5 & 6 & 7 \\
\hline (I) Aprender novas maneiras de pensar me empolga muito. & 1 & 2 & 3 & 4 & 5 & 6 & 7 \\
\hline (m) Prefiro minha vida repleta de enigmas para resolver. & 1 & 2 & 3 & 4 & 5 & 6 & 7 \\
\hline (n) Pensar abstratamente me atrai. & 1 & 2 & 3 & 4 & 5 & 6 & 7 \\
\hline $\begin{array}{l}\text { (o) Prefiro uma tarefa intelectual, difícil e importante a } \\
\text { uma outra que seja importante, mas que não me obrigue a } \\
\text { refletir muito. }\end{array}$ & 1 & 2 & 3 & 4 & 5 & 6 & 7 \\
\hline $\begin{array}{l}\text { (p) Sinto alívio em vez de satisfação depois de completar } \\
\text { uma tarefa que requereu grande esforço mental. }\end{array}$ & 1 & 2 & 3 & 4 & 5 & 6 & 7 \\
\hline $\begin{array}{l}\text { (q) É suficiente para mim que o trabalho tenha sido feito, } \\
\text { não me importa como e porquê foi feito. }\end{array}$ & 1 & 2 & 3 & 4 & 5 & 6 & 7 \\
\hline $\begin{array}{l}\text { (r) Usualmente não costumo opinar sobre questões quando } \\
\text { estas não me afetam pessoalmente. }\end{array}$ & 1 & 2 & 3 & 4 & 5 & 6 & 7 \\
\hline
\end{tabular}




\section{Análise dos dados}

\section{Mensuração da presença de heurísticas}

O nível de presença de heurística, objeto do bloco 1 do instrumento de pesquisa, foi mensurado atribuindo-se 1 (um) ponto para as alternativas com heurísticas inseridas e 0 (zero) ponto para as alternativas sem heurísticas.

Tabela 2: Composição da amostra por nível de heurística

\begin{tabular}{ccccc}
\hline & & Frequência & Percentual & Percentual acumulado \\
\hline \multirow{2}{*}{ Nível } & -3 & 5 & 3,9 & 3,9 \\
& -2 & 16 & 12,5 & 16,4 \\
-1 & 19 & 14,8 & 31,3 \\
0 & 32 & 25,0 & 56,3 \\
1 & 30 & 23,4 & 79,7 \\
2 & 19 & 14,8 & 94,5 \\
3 & 7 & 5,5 & 100,0 \\
Total & 128 & 100,0 & \\
\hline
\end{tabular}

Fonte: Elaboração própria (2011)

Para análise dos dados, em variável binária, os respondentes com níveis negativos receberam valor 0 (zero), menor presença de heurística, e os respondentes com níveis positivos receberam valor 1 (um), maior presença de heurísticas.

De acordo com a Tabela 2, 44\% dos respondentes apresentaram maior presença de heurísticas nas respostas aos cenários apresentados; já 31\% da amostra apresentou menor presença de heurísticas; 32 respondentes foram expurgados da análise, por apresentarem nível de heurística igual a 0 (zero). Esses resultados estão em consonância com os trabalhos desenvolvidos por Kahneman e Tversky (1979), que indicam que, diante de determinados cenários, as pessoas tendem a utilizar simplificações mentais, distorcendo, dessa forma, o processo de tomada de decisão. Além disso, esse resultado reafirma os achados de Buss (1995) quando se verifica que os respondentes utilizam atalhos mentais para organizar, avaliar e acompanhar atividades contábeis e financeiras.

\section{Validação do teste NFC}

Dentre as várias técnicas multivariadas, tem-se a análise fatorial. Segundo Aranha e Zambaldi (2008), essa é uma técnica estatística cujo escopo é caracterizar um conjunto de variáveis diretamente mensuráveis, chamadas de variáveis observadas, como a manifestação visível de um conjunto menor de variáveis hipotéticas e latentes, denominadas fatores comuns, e de um conjunto de fatores únicos, cada um deles atuando apenas sobre uma das variáveis observadas. O uso dessa técnica propicia duas funções principais: resumir e reduzir dados (Hair et al., 1998; Malhotra, 2001). Outra característica da análise é sua capacidade de determinar o grau de influência de determinada variável na explicação de um fator (posteriormente descoberto), além de analisar a intensidade das relações entre as variáveis observadas e, a partir delas, estimar um modelo fatorial subjacente capaz de reproduzir essas relações. 
Segundo Hair et al., valores de $\mathrm{KMO}$ abaixo de 0,50 são considerados inaceitáveis, entre 0,50 e 0,69, aceitáveis, e, acima de 0,70, são considerados desejáveis. O resultado desse teste apresentou o valor de 0,887, configurando-se como desejável.

O Teste de Esfericidade de Bartlett confirma a validade da aplicação da análise fatorial a um conjunto de variáveis, e é empregado para testar hipótese nula de que os itens na matriz de correlação não estão correlacionados, sugerindo que a análise fatorial da escala é apropriada. Para isso, seu nível de significância deve ser baixo o suficiente (inferior a 5\%) para rejeitar a hipótese nula e indicar que há uma relação intensa entre os itens (Hair et al., 1998). Os resultados da análise apresentaram nível de significância baixo o suficiente para rejeitar a hipótese nula e indicar que há uma relação forte entre os itens (Qui-quadrado igual a 665,639 e nível de significância igual a 0,000), além de legitimar o fato de a análise fatorial da escala poder ser considerada apropriada. A análise fatorial dos resultados indicou a existência de quatro fatores, conforme a Tabela 3.

Tabela 3. Análise de componentes principais - NFC

\begin{tabular}{ccccccc}
\hline \multirow{2}{*}{ Componente } & \multicolumn{5}{c}{ Autovalor inicial } & \multicolumn{3}{c}{ Somas extraídas dos carregamentos quadráticos } \\
\cline { 2 - 6 } & Total & \% da Variância & \% acumulado & Total & \% da Variância & \% acumulado \\
\hline 1 & 6,058 & 33,655 & 33,655 & 6,058 & 33,655 & 33,655 \\
2 & 1,655 & 9,192 & 42,847 & 1,655 & 9,192 & 42,847 \\
3 & 1,284 & 7,132 & 49,979 & 1,284 & 7,132 & 49,979 \\
4 & 1,163 & 6,464 & 56,443 & 1,163 & 6,464 & 56,443 \\
5 &, 999 & 5,548 & 61,991 & & & \\
6 &, 926 & 5,145 & 67,136 & & & \\
7 &, 767 & 4,262 & 71,398 & & & \\
8 &, 739 & 4,104 & 75,502 & & & \\
9 &, 679 & 3,770 & 79,272 & & & \\
10 &, 582 & 3,234 & 82,505 & & \\
11 &, 541 & 3,006 & 85,511 & & \\
12 &, 506 & 2,812 & 88,324 & & \\
13 &, 468 & 2,598 & 90,922 & & \\
14 &, 409 & 2,270 & 93,192 & & \\
15 &, 372 & 2,066 & 95,257 & & \\
16 &, 299 & 1,659 & 96,916 & & \\
17 &, 280 & 1,554 & 98,471 & & & \\
18 &, 275 & 1,529 & 100,000 & & & \\
\hline
\end{tabular}

Fonte: Elaboração própria (2011)

De acordo com Cattell (1996), Menezes (2006) e Shimada, Chiusoli e Mesetti (2010), o número de fatores deve ser estabelecido seguindo três critérios de retenção em uma análise.

O primeiro critério aplicado para determinar o número de fatores retido na análise foi o critério de Kaiser. A proposta é considerar apenas autovalores maiores que 1,0. A análise de autovalores indicou a presença de autovalor maior que 1 apenas nos quatro primeiros componentes, indicando a existência de quatro dimensões distintas para o bloco 1 desta pesquisa.

O segundo critério aplicado foi a proporção da variância. O autovalor acima de 1 é apenas um dos critérios para configuração de um fator, e é necessário notar a contribuição desses fatores na variância do autovalor inicial. Percebe-se que os fatores $2(1,655), 3(1,284)$ e $4(1,163)$, apesar de apresentarem autovalor acima de 1, não contribuem de forma tão significativa quanto o fator $1(6,058)$. 
O terceiro critério aplicado para determinar o número de fatores foi a análise do scree-plot, representado na Figura 2, que mostrou um ponto de inflexão entre os autovalores acima do ponto de ruptura da queda da curva da função após o fator 2 .

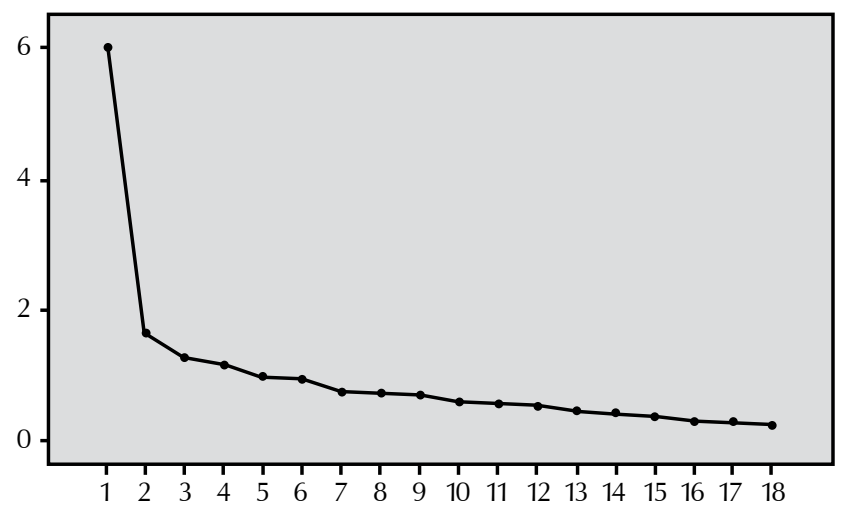

Figura 2. Scree-plot - NFC

Fonte: Elaboração própria (2011)

Decidiu-se, então, não considerar os fatores 3 e 4 . Uma nova análise de componentes principais foi gerada, dessa vez, solicitando a apresentação de somente dois fatores, conforme a Tabela 4.

Tabela 4: Análise de componentes principais com dois fatores - NFC

\begin{tabular}{ccccccc}
\hline \multirow{2}{*}{ Componente } & \multicolumn{2}{c}{ Autovalor inicial } & \multicolumn{3}{c}{ Somas extraídas dos carregamentos quadráticos } \\
\hline & Total & \% da Variância & \% acumulado & Total & \% da Variância & \% acumulado \\
\hline 1 & 6,058 & 33,655 & 33,655 & 6,058 & 33,655 & 33,655 \\
2 & 1,655 & 9,192 & 42,847 & 1,655 & 9,192 & 42,847 \\
3 & 1,284 & 7,132 & 49,979 & & & \\
4 & 1,163 & 6,464 & 56,443 & & & \\
5 &, 999 & 5,548 & 61,991 & & & \\
6 &, 926 & 5,145 & 67,136 & & & \\
7 &, 767 & 4,262 & 71,398 & & & \\
8 &, 739 & 4,104 & 75,502 & & & \\
9 &, 679 & 3,770 & 79,272 & & & \\
10 &, 582 & 3,234 & 82,505 & & & \\
11 &, 541 & 3,006 & 85,511 & & \\
12 &, 506 & 2,812 & 88,324 & & \\
13 &, 468 & 2,598 & 90,922 & & \\
14 &, 409 & 2,270 & 93,192 & & \\
15 &, 372 & 2,066 & 95,257 & & \\
16 &, 299 & 1,659 & 96,916 & & \\
17 &, 280 & 1,554 & 98,471 & & \\
18 &, 275 & 1,529 & 100,000 & & \\
\hline
\end{tabular}

Fonte: Elaboração própria (2011)

A partir dos critérios estabelecidos pelo método sugerido por Cattell (1966), Menezes (2006) e Shimada, Chiusoli e Messeti (2010), a análise dos componentes principais indicou a existência de duas dimensões distintas para o bloco 2 desta pesquisa. 
Tabela 5. Análise dos coeficientes de correlação - método pattern matrix

\begin{tabular}{lcc}
\hline & \multicolumn{2}{c}{ Componente } \\
& 1 & 2 \\
\hline P1 &, 420 &, 280 \\
P2 &, 760 &,- 061 \\
P3 &, 513 &, 248 \\
P4 &, 511 &, 244 \\
P5 &, 391 &, 260 \\
P6 &, 577 &, 132 \\
P7 &, 654 &, 126 \\
P8 &, 415 &, 045 \\
P9 &, 348 &, 350 \\
P10 &, 678 &,- 008 \\
P11 &, 707 &,- 224 \\
P12 &, 744 &,- 167 \\
P13 &, 642 &, 004 \\
P14 &, 636 &, 105 \\
P15 &, 738 &,- 079 \\
P16 &, 673 &,- 138 \\
P17 &,- 131 &, 780 \\
P18 &,- 207 &, 800 \\
\hline Fon & & \\
\hline
\end{tabular}

Fonte: Elaboração própria (2011)

Utilizou-se o método de rotação oblíqua Promax para a análise dos coeficientes de correlação, pois espera-se que, teoricamente, os itens estejam correlacionados. A rotação promax fornece, além da matriz fatorial, duas matrizes: a pattern matrix e a structure matrix, que delineiam os padrões oblíquos ou os clusters de intercorrelações entre as variáveis. As cargas fatoriais determinarão, dessa forma, os padrões e o grau de envolvimento de cada variável com os padrões. Enquanto a pattern matrix exibe quais variáveis estão altamente envolvidas em termos de cargas fatoriais em cada cluster, a structure matrix mostra a medida da correlação das variáveis com os padrões, como um todo.

Segundo Menezes (2006), a determinação da fatorial somente chega ao fim quando é examinada a afinidade dos itens com os fatores do modelo. Os constructos de um modelo fatorial são expurgados de acordo com os seguintes critérios: 1ํ) quando o valor absoluto da carga fatorial principal do item é menor do que $0,32,2^{\circ}$ ) quando existem cargas fatoriais similares em dois ou mais fatores em um mesmo item (a diferença entre os valores absolutos das cargas fatoriais dos itens é menor do que 0,10 ), e $3^{\circ}$ ) ausência de similaridade entre o conteúdo do item e o domínio teórico do construto.

A partir desses critérios, conforme ilustrado na Tabela 5, o item P9 (Gosto de tarefas que requerem pouco pensar, uma vez que as tenha aprendido) foi excluído, já que apresentou diferença inferior a 0,10 entre as cargas fatoriais nos fatores 1 e 2 . Os itens P17 (É suficiente para mim que o trabalho tenha sido feito, não me importa como e porquê foi feito) e P18 (Usualmente não costumo opinar sobre questões quando estas não me afetam pessoalmente) também foram excluídos, já que uma dimensão é formada pelo agrupamento de 3 ou mais fatores, conforme recomendação de Pasquali (1998) e Hair et al. (1998), configurando, assim, que os elementos da NFC apresentaram contato unidimensional, já que o fator 2 foi composto somente por 2 itens. A dimensão desse fator revela, de acordo com os itens agrupados no fator 1 , a necessidade do respondente em práticas cognitivas.

De acordo com Pasquali, um dos pontos mais críticos na elaboração dos instrumentos psicológicos é a questão da dimensionalidade. Visto que o item deve adotar o pressuposto de unidimensionalidade, isto é, referir-se a um único traço latente, a grande dificuldade da Psicologia consiste em determinar a estrutura interna, semântica dos fatores que estuda, pois as próprias teorias psicológicas acabam por não oferecer facilidade e clareza conceituais para transformar definições constitutivas em definições operacionais.

O primeiro e único construto conglomera os conceitos mais importantes do Need 
For Cognition, tanto que foi o que mais agregou variáveis nessa escala; o que ficou de fora foi expurgado por critérios estatísticos de dimensionalidade de um fator. Esse fator aponta a aproximação dos respondentes em refletir, ponderar sobre determinada situação, e refletir é pensar arduamente, é buscar esclarecer ideias e analisar pensamentos.

Após a exclusão dos três itens do Bloco 2 desta pesquisa, torna-se necessário analisar os 15 itens restantes, a partir dos critérios de dimensionalidade, confiabilidade e convergência.

A análise de dimensionalidade foi realizada por meio do uso de análise fatorial, mediante emprego da técnica de análise de componentes principais e uso do Índice KMO e Teste de Esfericidade de Bartlett. A existência de um único autovalor (com valor superior a 1) certifica a unidimensionalidade da escala observada. O resultado do teste $\mathrm{KMO}$ apresentou o valor de 0,893 , configurando-se como desejável. O resultado do Teste de Esfericidade de Bartlett apresentou um nível de significância baixo o suficiente para rejeitar a hipótese nula e indicar que há uma relação forte entre os itens (Qui-quadrado igual a 691,835 e nível de significância igual a 0,000).

Para analisar a confiabilidade, foi utilizado o alfa de Cronbach, sendo considerado que um nível mínimo de confiabilidade geralmente aceito é 0,7, apesar de ser reduzido para 0,6 em pesquisas exploratórias (Hair et al., 1998). Assim, o resultado desse teste (alfa de Cronbach igual a 0,879) atesta a confiabilidade da escala utilizada. Vale destacar que tal teste é dependente do $n$, e pode ser significativo mesmo quando as correlações são muito baixas.

Por fim, a análise de convergência foi realizada por meio da análise do coeficiente de Pearson. Observou-se que todos os coeficientes foram positivos e significativos (alguns no nível de 1\% e outros no nível de 5\%), indicando a existência de uma forte correlação nos itens da escala, o que sugere a possibilidade do uso de média para os itens da escala.

\section{Mensuração do nível de necessidade de cognição}

Foi adotado o uso do teste de uma amostra para a média a cada output do NFC. Optou-se por comparar a média da escala com o valor de 7 (sete), ponto máximo da escala, pois o envolvimento absoluto com práticas cognitivas deveria fazer com que as respostas fossem iguais a 7 (sete), e um envolvimento impreciso faria com que as respostas se afastassem da constante 7 . Portanto,

Tabela 6: Estatísticas descritivas NFC

\begin{tabular}{lccccc}
\hline Escala & N & Mínimo & Máximo & Média & Desvio Padrão \\
\hline NFC & 128 & 1,00 & 7,00 & 4,9577 & 1,34219 \\
N válido & 128 & & & & \\
\hline
\end{tabular}

Fonte: Elaboração própria (2011)

quanto mais a média se distanciar de 1 (na escala de 1 a 7) maior será o envolvimento do respondente. A Tabela 6 aponta as médias relacionadas à escala utilizada para a verificação do nível de necessidade de cognição dos respondentes. Pode ser observado que a média se aproxima do ponto máximo, queé 7 (4,9577). 
A partir dessa média, com o cruzamento dos dados fornecidos através da variável membership, fornecida pela análise de cluster através do método não hierárquico, obteve-se a Tabela 7, a seguir.

Tabela 7. Identificação do cluster NFC

\begin{tabular}{|c|c|c|}
\hline $\begin{array}{l}\text { Identificação do } \\
\text { cluster }\end{array}$ & & $\begin{array}{l}\text { Escala } \\
\text { NFC }\end{array}$ \\
\hline Baixo NFC (0) & $\begin{array}{l}\text { Média } \\
\mathrm{N} \\
\text { Desvio Padrão }\end{array}$ & $\begin{array}{l}3,6487 \\
59 \\
1,0942\end{array}$ \\
\hline Alto NFC (1) & $\begin{array}{l}\text { Média } \\
\mathrm{N} \\
\text { Desvio Padrão }\end{array}$ & $\begin{array}{l}5,9881 \\
69\end{array}$ \\
\hline Total & $\begin{array}{l}\text { Média } \\
\mathrm{N} \\
\text { Desvio Padrão }\end{array}$ & $\begin{array}{l}4,8005 \\
128 \\
1,07294\end{array}$ \\
\hline
\end{tabular}

Fonte: Elaboração própria (2011)

De acordo com a Tabela 7, 46\% dos respondentes apresentaram baixa necessidade de cognição, enquanto 69 alunos dos 128 pesquisados apresentaram alta necessidade de cognição.

Na escala analisada, os que possuíam alto NFC apresentaram média igual a 5,9881, quase 1,05 pontos acima da média geral apresentada na Tabela 6, enquanto os que possuíam baixo NFC apresentaram aproximadamente 1,3 pontos abaixo da média geral $(3,6487)$. Os clusters da Tabela 7 serão utilizados nos testes de hipóteses para comparar o comportamento entre os que demonstraram baixa necessidade de cognição e os que apresentaram alta necessidade de cognição.

\section{Resultados dos testes de hipóteses}

Com o objetivo de testar a hipótese de existência de associação do nível de necessidade de cognição com relação aos níveis de presença de heurística, foi inicialmente ajustado um modelo logístico simples que continha, como variável, a resposta "a presença de heurística", e, como variável independente, a variável envolvida na análise, a NFC.

Inicialmente, foi considerado um modelo logístico binário em que $p(x)$, a probabilidade de a variável resposta sendo igual a 1 (um), ou seja, de o indivíduo apresentar maior presença de heurística dado o valor da variável independente, sendo esse modelo definido pela Equação 1.

$$
\log \left\{\frac{p(x)}{1-p(x)}\right\}=\beta_{0}+\beta_{1}+x, \text { em que }
$$

Equação 1

$X$ : representa o valor da variável necessidade de exercer práticas cognitivas;

$\beta_{0}:$ o intercepto;

$\beta_{1}$ : parâmetro desconhecido associado à covariável $X$ 
A proposta da hipótese $\mathrm{H}_{1}$ foi avaliar se quanto menor for o nível de necessidade de cognição, maior será a presença de heurísticas. Com essa finalidade, foram formuladas as seguintes hipóteses, nula e alternativa, respectivamente.

$\mathrm{H}_{1.0}$ : Não existe relação entre os níveis de necessidade de cognição, com a presença de heurísticas; $\mathrm{H}_{1.1}$ : Quanto menor for o nível de necessidade de cognição, maior será a presença de heurísticas. Da análise da Tabela 8, pode-se concluir que a variável NFC apresentou efeito significativo em relação à resposta, dado que o p-valor foi menor que o nível de significância $(0,001)$, logo, a hipótese de nulidade $\left(\mathrm{H}_{1.0}\right)$ foi rejeitada.

Quanto à interpretação desses coeficientes, entende-se que, a cada aumento do escore NFC, a chance de apresentar maior heurística é 0,98 vezes maior do que de apresentar menor viés cognitivo, ou seja, a chance de um indivíduo com baixa necessidade de cognição apresentar heurísticas é quase o dobro do que apresentam os indivíduos com alta necessidade de cognição, corroborando assim os estudos de Cohen, Stotland e Wolfe (1955) e Cacioppo e Petty (1982).

Tabela 8. Avaliação bivariada entre os fatores de risco e a presença de heurística

\section{Heurísticas}

\begin{tabular}{lcc} 
Fatores de risco & OR [IC 95\%] & P-valor \\
\hline NFC & $0.98(0.68 ; 1.28)$ & 0.001 \\
\hline
\end{tabular}

Fonte: Elaboração própria (2011)

Outra ferramenta foi utilizada a fim de corroborar os resultados encontrados, o Teste Não Paramétrico de Mann Whitney, para avaliar possíveis diferenças entre os grupos que apresentam menor e maior presença de heurística em relação às variáveis criadas a partir da análise fatorial. Na Tabela 8, estão descritos os resultados desse teste, que revelam que os grupos com menor e maior heurísticas são diferentes em relação ao escore necessidade de exercer práticas cognitivas, dado que o p-valor do teste foi menor que o nível de significância de 5\%. Esse teste corrobora os resultados já apontados na Tabela 8.

Tabela 9. Avaliação dos fatores de risco em relação aos níveis de presença de heurística

\begin{tabular}{lccc}
\hline \multirow{2}{*}{ Fatores de risco } & \multicolumn{2}{c}{ Heurísticas } & P-valor* \\
& Menor presença & Maior presença & \\
\hline NFC & 0,189 & $-0,223$ & 0.001 \\
\hline * Teste de Mann Whitney & & Fonte: Elaboração própria (2011)
\end{tabular}

Sob a ótica do modelo múltiplo, a Equação 2 se diferencia apenas pela inclusão no modelo de outras variáveis relevantes para o estudo, sendo estas analisadas no modelo. Diante dos resultados encontrados na Tabela 5, foi então ajustado um modelo logístico múltiplo com as variáveis envolvidas, proposto de tal forma: 


$$
\log \left\{\frac{p(x)}{1-p(x)}\right\}=\beta_{0}+\beta_{2 a}+X_{2 a}, \text { em que }
$$

\section{Equação 2}

$X_{2 a}$ : representa o valor da variável necessidade de exercer práticas cognitivas;

$\beta_{0}:$ o intercepto;

$\beta_{2 a}$ : parâmetro desconhecido associado à covariável $X_{2 \mathrm{a}}$;

Com isso, a Tabela 10 apresenta evidências de que a variável NFC continua expondo efeito significativo em relação à presença de heurística ( $p$-valor igual a 0,01).

Tabela 10. Avaliação múltipla dos fatores de risco na presença de heurística

\begin{tabular}{lcc}
\hline & \multicolumn{2}{c}{ Heurísticas } \\
Fatores de risco & OR [IC 95\%] & P-valor \\
\hline NFC & $0.61(0.41 ; 0.98)$ & 0.010 \\
\hline
\end{tabular}

Fonte: Elaboração própria (2011)

\section{Conclusão}

Este estudo teve como objetivo validar a escala Need For Cognition (NFC) em estudos em Contabilidade comportamental. Ademais, buscou medir possíveis correlações entre o nível de necessidade de cognição e a existência de vieses cognitivos em decisões contábeis e financeiras. Para efetivar o processo de validação completo, foram realizadas duas validações - critério e construto. Para a validação do NFC, foi utilizada a analise fatorial, em que os índices de dimensionalidade, confiabilidade e convergência apresentaram-se desejáveis e significativos. A partir dos critérios adotados na analise fatorial, dos 18 outputs do NFC, três outputs foram excluídos, e os demais exibiram-se segregados no fator 1, que foi denominado "necessidade do respondente em práticas cognitivas", configurando assim, uma escala unidimensional.

Para responder ao objetivo secundário deste estudo, foram construídos dois blocos de pesquisa com situações que envolviam alguns conceitos relevantes para o trabalho: (a) heurísticas e (b) NFC. A construção desses cenários visou a observar a ocorrência de três heurísticas abordadas: (a) ancoragem, (b) representatividade e (c) disponibilidade de instâncias, observando o quanto as variáveis independentes deste estudo explicavam a ocorrência desses fenômenos em um único contexto: a presença de heurísticas.

Os testes empíricos realizados confirmaram a hipótese do efeito das heurísticas em todas as perguntas do questionário e corroboraram, portanto, os resultados obtidos em outras pesquisas nessa mesma linha.

Este estudo mostrou que existe relação significativa entre o nível de necessidade de cognição e a presença de heurísticas, o que vai de encontro à pesquisa de Fleischhauer et al. (2010), que 
concluíram que a inteligência de um adulto não pode ser plenamente compreendida sem considerar fatores cognitivos, e que vieses e nível de cognição são grandezas inversamente proporcionais.

Respondendo ao problema de pesquisa proposto, na metodologia conduzida neste estudo, foi observado o impacto do nível de cognição na maximização da ocorrência de heurísticas em decisões gerenciais; concluise que, a cada aumento do escore NFC, a chance de apresentar maior heurística é 0,98 vezes maior do que de apresentar menor viés cognitivo, ou seja, a chance de um indivíduo com baixa necessidade de cognição apresentar heurísticas é quase o dobro do que apresentam os indivíduos com alta necessidade de cognição.

Algumas limitações importantes do estudo merecem atenção. Em primeiro lugar, conforme discutido na exposição dos procedimentos metodológicos, a amostra utilizada obedeceu a critérios de conveniência, em função do arcabouço do quase-experimento, não havendo a designação aleatória dos participantes entre as condições das variáveis relativas ao perfil do respondente. Novas pesquisas podem buscar inserir outras variáveis ao modelo proposto para testar essas relações. Dessa forma, acredita-se que este seja um tema fecundo para o desenvolvimento de novas pesquisas, sendo que este estudo oferece a validação de um instrumento psicométrico em Contabilidade comportamental, além de uma pequena contribuição para o desenvolvimento de outras investigações relacionadas ao tema.

Portanto, fica evidenciada, neste trabalho, a importância da discussão acerca da Contabilidade comportamental, para que seu desenvolvimento possa fazer com que sejam resolvidos problemas que incidem em decisões gerenciais quando não notados os aspectos cognitivos e psicológicos de quem efetivamente toma decisões.

Raimundo Nonato Lima Filho

Doutorando em Administração pela Universidade Federal da Bahia e professor da Universidade do Estado da Bahia, Senhor do Bonfim - BA - Brasil.

E-mail: rnfilho@uneb.br

Adriano Leal Bruni

Doutor em Administração pela Universidade de São Paulo e professor da Faculdade de Ciências Contábeis da Universidade Federal da Bahia, Salvador - BA - Brasil.

E-mail: albruni@ufba.br

Igor Gomes Menezes

Doutor em Psicologia pela Universidade Federal da Bahia e professor da Universidade Federal da Bahia, Salvador - BA - Brasil.

E-mail: igorgmenezes@gmail.com

Endereço para envio de correspondência:

Universidade do Estado da Bahia - Departamento de Educação VII. Rodovia Lomanto Júnior, BR 407, Km 127. CEP: 48970-000. Senhor do Bonfim, BA. 
Referências
Aranha, F., \& Zambaldi, F. (2008). Análise Fatorial em Administração. São Paulo: Cengage Learning.

Axsom, D., Yates, S., \& Chaiken, S. (1987). Audience response as a heuristic cue in persuasion. Journal of Personality and Social Psychology, 53, 30-40.

Cacioppo, J. P., \& Petty, R. E. (1982). The need for cognition. Journal of Personality and Social Psychology, 42(1), 116-131.

Cacioppo, J. T., Petty, R. E., \& Kao, C. F. (1984). The efficient assessment of need for cognition. Journal of Personality Assessment, 48(3), 306-307.

Cacioppo, J. P., Petty, R. E., Feinstein, J., \& Jarvis, W. B. G. (1996). Dispositional differences in cognitive motivation: The life and times of individuals varying in need for cognition. Psychological Bulletin, 119, 197-253.

Cacioppo, J. P., Petty, R. E., \& Morris K. J. (1983). Effects of need for cognition on message evaluation, recall, and persuasion. Journal of Personality and Social Psychology, 45(4), 805-818.

Cacioppo, J. P., Petty, R. E., Kao, C. F., \& Rodriguez, R. (1986). Central and peripheral routes to persuasion: An individual difference perspective. Journal of Personality and Social Psychology, 51, 1032-1043

Cacioppo, J. P., \& Petty, R. E. (1984). The need for cognition: Relationship to attitudinal processes. In Interfaces in psychology: Social perception in clinical and counselling psychology (Vol. 2). Lubbock, Texas: Texas Tech University Press.

Cattell, R. B. (1966). The scree test for the number of factors. Multivariate Behavioral Research, 1, 245-267.

Chaiken, S. (1987). The heuristic model of persuasion. In M. P. Zanna, J. Olson \& C. P. Herman (Eds.). Social influence: The Ontario Symposium (Vol. 5). Hillsdale, NJ: Lawrence Erlbaum Associates.

Cohen, A. R., Stotland, E., \& Wolfe, D. M. (1955). An experimental investigation of need for cognition. Journal of Abnormal and Social Psychology, 51, 291-294.

Cooper, D. R., \& Schindler, P. S. (2003). Métodos de pesquisa em administração. Porto Alegre: Bookman.

Deliza, R., Rosenthal, A., \& Costa, M. C. da. (2003). Tradução e validação para a língua portuguesa de questionário utilizado em estudos de consumidor. Ciência e Tecnologia de Alimentos, 23(1), 43-48.

Elliott, W. B., F. Hodge, J., Kennedy, \& Pronk, M. (2007). Are MBA students a good output for nonprofessional investors? The Accounting Review, 139-168.

Fenwick, W. E., \& Cheryl, L. B. (2008). An exploration of administrative heuristics in the United States and the United Kingdom. Journal of School Leadership, 18(1), 96-119.

Fleischhauer, M., Enge S., Brocke, B., Ullrick, J., Strobel A., \& Strobel, A. (2010). Same or different? Clarifying the relationship of need for cognition to personality and intelligence. Personality and Social Psychology Bulletin, 36(1), 82-96.

Hair, J., Anderson, R., Tatham, R., \& Black, W. (1998). Multivariate data analysis (5nd. ed.). Upper Saddle River: Prentice-Hall.

Hansen, D. E., \& Helgeson, J. G. (1996). The effects of statistical training on choice heuristics in choice under uncertainty.
Journal of Behavior Decision Making, 9, 41-57.

Haugtvedt, C. P., Petty, R. E., \& Cacioppo, J. T. (1992). Need for cognition and advertising: Understanding the role of personality variables in consumer behavior. Journal of Consumer Psychology, 1(3), 239- 260.

Jeon, K. Y., \& Shyam, S. S. (2010). Heuristic versus systematic processing of specialist versus generalist sources in online media. Human Communication Research, 36(2), 103-124.

Kahneman, D., \& Riepe, M. W. (1998). Aspects of investor psychology. Journal of Portfólio Management, 24, 52-65.

Kahneman, D., \& Tversky, A. (1979). Prospect theory: An analysis of decision under risk. Econometrica, 47, 263-291.

Kivetz, R. (1999). Advances in research on mental accounting and reason-based choice. Marketing Letters. Springer Netherlands, 10(3), 249-266.

Liyanarachchi, G. A., \& Milne, M. J. (2005). Comparing the investment decisions of accounting practitioners and students: An empirical study on the adequacy of student surrogates. Accounting Fórum, 29, 121-35

Malhotra, N. K. (2001). Pesquisa de marketing: uma orientação aplicada. Porto Alegre: Bookman.

Meyer, P. L. (1983). Probabilidade: aplicações à estatística (2a. ed.). Rio de Janeiro: Livros Técnicos e Científicos.

Menezes, I. G. (2006). Escala de Intenções Comportamentais de Comprometimento Organizacional (EicCo): concepção, desenvolvimento, validação e padronização. Dissertação de mestrado em Psicologia. PPGPSI. Universidade Federal da Bahia - UFBA, Salvador, BA.

Milanez, F. (2003). Desenvolvimento sustentável. In A. D. Cattani (Org.). A outra economia. Porto Alegre: Veraz Editores.

Pasquali, L. (1998). Histórico dos instrumentos psicológicos. In L. Pasquali (Org.). Instrumentos psicológicos: Manual Prático de Elaboração. Brasília, DF: Editora UnB.

Pohl, R. F. (2006). Empirical tests of the recognition heuristic. Journal of Behavior Decision Making, 19(3), 251-271.

Raymundo, V. P. (2009). Construção e validação de instrumentos: um desafio para a psicolinguística. Letras de Hoje, 44(3), 86-93.

Shimada, A. T., Chiusoli, C. L., \& Messetti, A. V. L. (2010). Análise fatorial: Avaliação de estabelecimentos alimentícios. In XIII Seminários em Administração, Anais do XIII SEMEAD, São Paulo, SP.

Simon, H. A. (1955). A behavioral model of rational choice. Quarterly Journal of Economics, 69, 99-118.

Subhashis, M., Subhas, N., \& Bhargab, B. B. (2010). Separating multi-color points on a plane with fewest axis-parallel lines. Fundamenta Informaticae, 99(3), 315-324.

Tversky, A., \& Kahneman, D. (1974). Judgment under uncertainty: Heuristics and biases. Science, 185, 1124-1131.

Verplanken, B., Hazenberg, P. T., \& Palenewen, G. R. (1992). Need for cognition and external information search effort. Journal of Research in Personality, 26, 128-136. 